\title{
Exposición selectiva y partidismo de las audiencias en España. El consumo de información política durante las campañas electorales de 2008 y $2011^{1}$
}

María Luisa Humanes²

Recibido: 2013-11-09

Envío a pares: 2013-11-12
Aprobado por pares: 2014-02-05

Aceptado: 2014-02-20

DOI: 10.5294/pacla.2014.17.3.9

\section{Para citar este artículo / To reference this article / Para citar este artigo}

Humanes, M. L. Septiembre de 2014. Exposición selectiva y partidismo de las audiencias en España. El consumo de información política durante las campañas electorales de 2008 y 2011. Palabra Clave 17 (3), 773-802. DOI: 10.5294/pacla.2014.17.3.9

\section{Resumen}

Lazarsfeld, Berelson y Gaudet (1948) apuntaron que los efectos de los medios se tamizan por los procesos de selección, atención y retención, que actúan a favor del refuerzo de las predisposiciones previas de los individuos cuando se enfrentan a los contenidos de los medios. Actualmente asistimos a un resurgir de las propuestas que vuelven a afirmar los efectos mínimos de los medios y la pervivencia de la teoría de la exposición selectiva, naturalmente adaptándola a la nuevas situaciones de consumo mediático. El estudio aborda si los españoles se exponen selectivamente a la información política en periodos de campaña electoral y si ello se corresponde con un comportamiento partidista de las audiencias. Se han utilizado las encuestas postelectorales del Centro de Investigaciones Sociológicas de 2008 y 2011. La prensa es el medio de información política y electoral más condicionado por el efecto de la exposición selectiva provocada por las orientaciones políticas.

\footnotetext{
1 Este artículo forma parte del proyecto de investigación "Implantación de la TDT en España e impacto sobre el pluralismo”, financiado por el Ministerio de Ciencia e Innovación de España (ref. CS2010-17898/COMU).

2 Universidad Rey Juan Carlos, España. marialuisa.humanes@urjc.es
} 


\section{Palabras clave}

Exposición selectiva - partidismo - campaña electoral - información política - España (Fuente: Tesauro de la Unesco).

\section{Selective Exposure and Partisanship among Audiences in Spain. Consumption of Political Information during Election Campaigns: 2008 and 2011}

\section{Abstract}

According to Lazarsfeld, Berelson and Gaudet (1948), the effect of the media is limited by selection, attention and retention processes and individuals seek to reinforce their previous predispositions when selecting media outlets. We now see a resurgence of proposals that affirm, once again, the minimal effect of the media and survival of the theory of selective exposure, obviously adapted to the new circumstances of media consumption. The study examines whether or not the Spanish selectively expose themselves to political information during election campaign periods, and if doing so corresponds to partisan behavior by the audience. Post-election surveys conducted by the Centro de Investigaciones Sociológicas (Sociological Research Center) in 2008 and 2011 were used. The press is the political and electoral medium most affected by selective exposure based on political orientation.

\section{Keywords}

selective exposure, partisanship, electoral campaign, political news, Spain (Source: UNESCO Thesaurus). 


\section{Exposição seletiva e partidismo das audiências na Espanha. 0 consumo de informação política durante as campanhas eleitorais de 2008 e 2011}

\section{Resumo}

Lazarsfeld, Berelson e Gaudet (1948) assinalaram que os efeitos dos meios são depurados pelos processos de seleção, atenção e retenção, que atuam a favor do reforço das predisposições dos indivíduos quando se deparam com os conteúdos dos meios. Atualmente, assistimos a um ressurgir de propostas que afirmam os efeitos mínimos dos meios e da sobrevivência da teoria da exposição seletiva, naturalmente adaptando-a às novas situações de consumo mediático. $\mathrm{O}$ estudo aborda se os espanhóis se expõem seletivamente à informação política em períodos de campanha eleitoral e se isso se refere a um comportamento partidista das audiências. Foram utilizadas enquetes pós-eleitorais do Centro de Pesquisas Sociológicas de 2008 e 2011. A imprensa é o meio de informação política e eleitoral mais condicionado pelo efeito da exposição seletiva provocada pelas orientações políticas.

\section{Palavras-chave}

Exposição seletiva, partidismo, campanha eleitoral, informação política, Espanha (Fonte: Tesauro da Unesco). 


\section{Introducción}

En las dos últimas décadas ha aumentado el número de medios de comunicación a través de los cuales los españoles reciben información política. El número de las cadenas de radio y televisión, sobre todo, ha aumentado gracias a los sucesivos planes de concesiones de licencias a empresas privadas y de la puesta en funcionamiento de servicios de radiotelevisión públicos autonómicos. Además han proliferado con mayor o menor fortuna publicaciones online. Este panorama mediático permite por una parte una mayor capacidad de selección de las fuentes de información que cada ciudadano tiene a su disposición para informarse, y por otra la fragmentación de la audiencia para crear nichos en función de las predisposiciones ideológicas y políticas.

Esta situación ha sido ampliamente constatada en el ámbito anglosajón, en el que cadenas como la conservadora Fox han conseguido un considerable éxito empresarial basándose en un discurso periodístico partidista y polarizado, precisamente en la cuna del periodismo objetivo. Igualmente, la proliferación de publicaciones online ha contribuido a la fragmentación de las audiencias en función de sus ideas políticas. Esta situación ha provocado la recuperación de la teoría de la exposición selectiva propuesta originalmente por Lazarsfeld, Berelson y Gaudet (1948) para adaptarla a las nuevas formas de consumo mediático (Mutz, 2006; Bennett e Iyengar, 2008; Iyengar y Kahn, 2009; Stroud, 2010).

Este artículo se propone retomar esta teoría y sus nuevas versiones para corroborar empíricamente si los españoles se exponen selectivamente a la información política en periodos de campaña electoral, y si ello se corresponde con un comportamiento partidista de las audiencias.

\section{Exposición selectiva y exposición selectiva partidista}

La teoría de la exposición selectiva afirma que el consumo de los medios está determinado por las predisposiciones de la audiencia, que busca confirmar o reforzar sus opiniones en los contenidos de los medios. El 
estudio inicial de Lazarsfeld, Berelson y Gaudet (1948) apuntaba que los efectos de los medios se matizan por los procesos de selección, atención y retención, que actúan a favor del refuerzo de las predisposiciones y actitudes previas de los individuos cuando se enfrentan a los contenidos de los medios. A la selección, atención y retención selectivas se suman como factores mediadores los grupos de referencia, la comunicación interpersonal, los líderes de opinión y la naturaleza de los medios en el libre mercado (Kappler, 1960, p. 19). En esta primera fase se entiende, por lo tanto, que los ciudadanos buscarían en los contenidos mediáticos la mayor consonancia posible con sus ideas previas sobre la realidad (Stroud, 2010). Dado que la exposición es el paso previo para el resto de los procesos, el análisis de la exposición se convirtió en un tópico de investigación especialmente importante.

En los años sesenta se sucedieron las críticas a esta perspectiva (Stroud 2011). Autores como Freedman y Sears (1965) sugirieron que la correlación entre las opiniones sostenidas por los medios y las mantenidas por sus audiencias no se explican por el rechazo de éstas a las opiniones contrarias, sino porque el entorno mediático tiende a ofrecer a su audiencia más informaciones afines que disonantes (defacto selectivity). Las ideas de Freedman y Sears tuvieron un gran impacto, de modo que la teoría de la exposición selectiva cayó en desuso durante décadas. En la actualidad, los cambios en los sistemas de medios, es decir, la mayor disponibilidad de fuentes de información, rebaja los efectos del argumento de la selectividad de hecho.

Gracias a la teoría de la disonancia de Festinger, se reinterpretaron los postulados de la exposición selectiva, cambiando el centro de atención desde el concepto de rechazo a la información disonante hacia el de búsqueda (seeking) de la información (Frey, 1986, pp. 50 y ss.). Frey, en su revisión sobre las investigaciones realizadas con esta nueva perspectiva, destaca cinco condiciones bajo las cuales explicar la exposición selectiva: la capacidad de elección entre diversas fuentes de información, el compromiso del individuo con el tema de la información, la credibilidad de la fuente, la cantidad y tipo de la información entre la cual elegir y la intensidad de la disonancia cognitiva. 
Finalmente, la controversia entre partidarios y detractores de la exposición selectiva ha derivado en las últimas décadas en un renovado interés por analizar las condiciones que pueden favorecer la exposición selectiva y en concreto la selección orientada políticamente, de tal forma que la teoría de la exposición selectiva se ha colocado entre las ocho perspectivas más utilizadas en el siglo XXI (Bryant y Miron, 2004, p. 696). Bennett e Iyengar (2008) se preguntan si estamos ante un resurgir de los efectos mínimos de los medios debido entre otros factores a la multiplicación de medios de comunicación por los cuales recibir información. El crecimiento de Internet como fuente de búsqueda de información no ha hecho más que reforzar esta idea, dado que los ciudadanos aumentan su capacidad de encontrar información más fácilmente (Valentino et al., 2009). Por ejemplo, la customización de la búsqueda de información que permiten las webs de generación de contenidos enlaza con el proceso de exposición selectiva (Dylko y McCluskey, 2012, p. 261).

La recuperación de la idea de la exposición mediática motivada por las predisposiciones ha implicado el perfeccionamiento tanto de los planteamientos teóricos como de los diseños metodológicos. La rigurosa revisión de Stroud (2008) muestra el persistente debate entre quienes defienden la relación entre exposición y orientaciones y quienes matizan los resultados de estas investigaciones. En esta segunda etapa de la teoría de la exposición selectiva, Kinder (2003) y Zaller (1992) han seguido el camino marcado por los críticos al afirmar que la evidencia de que los individuos buscan siempre la información acorde con sus opiniones no es tan fuerte. D’Alessio y Allen (2002) en su metaanálisis de los estudios publicados entre 1956 y 1996 corroboraron esta idea de un moderado soporte $(r=0.22$, p <.001) a la hipótesis de la exposición selectiva.

No obstante, también se ha desarrollado una abundante literatura en apoyo de la teoría. Stroud (2011, pp. 15-21) utiliza cuatro aproximaciones que justifican la existencia de la exposición selectiva. La primera -ya citada- es la teoría de la disonancia cognitiva. En relación con ésta, la autora cita las perspectivas sobre la motivaciones, que engolaba bajo el rotulo de 'motivations beyond dissonances'. En tercer término incluye la metáfora del avaro cognitivo, que afirma que la exposición selectiva se daría en mayor 
medida cuando el individuo presenta una limitada capacidad para procesar información. Por último, la percepción de la calidad de la información constituye una explicación de por qué ocurre la exposición selectiva.

La misma autora señala que una de las posibles explicaciones para entender la discrepancia entre los investigadores respecto a la validez de esta teoría es la cantidad de condiciones contingentes que afectan el proceso de exposición a los contenidos de los medios (Stroud, 2008). Nos encontraríamos en una situación semejante a la señalada por numerosos autores respecto a las investigaciones sobre el efecto agenda-setting (Humanes y Moreno, 2012). Una de las hipótesis expresa la posibilidad de que diferentes tipos de medios promuevan una mayor o menor tendencia al uso selectivo. En nuestro trabajo, la hipótesis 2 plantea precisamente que la exposición a la información sobre la campaña electoral a través de los diarios se verá más orientada por las predisposiciones políticas que en los medios audiovisuales. Stroud (2008) encontró en su estudio sobre la campaña presidencial de 2004 que la elección de la televisión por cable está más determinada por las predisposiciones políticas que el resto de los medios. Goldman y Mutz (2011) también han confirmado esta hipótesis en su trabajo basado en una encuesta realizada en once países (2011, p. 51).

Otra de las variables consideradas en el estudio de la exposición selectiva es el tiempo. La mayoría de los estudios han considerado el uso de un tipo de medio o de formato en un momento concreto, pero resulta de gran interés analizar los posibles cambios en el desarrollo del proceso exposición, por ejemplo, a lo largo de una campaña electoral (Stroud, 2008; Matthes, 2012). Los estudios con encuesta panel ofrecerían de acuerdo a esta hipótesis mejor apoyo a la teoría que las investigaciones basadas en el método experimental (Mutz y Martin, 2001; Goldman y Mutz, 2011).

Otro de los determinantes de la exposición selectiva es el tópico sobre el cual se informa. Aunque los trabajos sobre exposición selectiva se han dedicado en su mayor parte a temas políticos, no son los únicos contenidos en los que se produce exposición selectiva. La conclusión es que diferentes contenidos afectan el grado de exposición selectiva (Stroud, 2006). 
Por último, las características del sistema de medios se han considerado un condicionante de la exposición selectiva, como han puesto de manifiesto Goldman y Mutz (2011).

Para comprender mejor el concepto de exposición selectiva resulta muy oportuno acudir a otros conceptos asociados con él. Diana Mutz ha abordado en sucesivos trabajos (Mutz y Martin, 2001; Goldman y Mutz, 2011) la idea de que los medios de comunicación pueden favorecer la selección de puntos de vista distintos a los propios. El concepto de crosscutting exposure se asocia al de exposición selectiva para analizar cómo los medios pueden facilitar o dificultar la elección de contenidos que no coinciden con las predisposiciones del individuo. La exposición a mensajes que no concuerdan con nuestras propias opiniones se ve favorecida bajo ciertas condiciones, sobre todo por la televisión más que por la prensa y por un sistema de medios en el que no se produzca paralelismo político. Los resultados de la encuesta en 11 países permiten generalizar que la naturaleza del sistema mediático facilita o inhibe la exposición selectiva y su contraparte: la exposición transversal (Goldman y Mutz, 2011, p. 56). Matthes (2012) ha encontrado evidencias del efecto de la exposición a la cobertura con presencia de distintas perspectivas sobre los votantes en distintos momentos de la campaña electoral.

Por otra parte, los investigadores que abordan la exposición selectiva a través de diseños experimentales han trabajado con el denominado hostile media effect (Vallone, Ross y Lepper, 1985). La ya mencionada fragmentación partidista del mercado televisivo en los Estados Unidos ha producido el incremento del número de individuos que tienden a percibir las noticias equilibradas como sesgadas si éstas no concuerdan con sus puntos de vista. Pfau, Houston y Semmler (2007) hipotetizaban en su estudio sobre los cambios en las campañas presidenciales en los Estados Unidos que la exposición selectiva tenía que acompañarse de los conceptos de hostilidad y desconfianza hacia los medios para explicar que los individuos consuman cada vez mensajes más partidistas, sobre todo a través de internet (2007, p. 33). 
Uno de los tópicos en los que se ha corroborado con más fuerza la tesis de la exposición selectiva ha sido su aplicación al consumo de información política. La hipótesis central sería que las personas seleccionan los contenidos mediáticos congruentes con sus predisposiciones políticas. Como hemos señalado al inicio, el avance de un periodismo menos objetivo y más sesgado políticamente ha multiplicado las evidencias de que la exposición selectiva partidista se estaría también incrementando (Iyengar y Kahn, 2009). La hipótesis del acuerdo anticipado afirma que los votantes prefieren rechazar la información que contraviene sus creencias y concentrarse en aquellas que las refuerzan (Iyengar et al., 2008). Stroud (2008) concluye en su estudio sobre la campaña electoral norteamericana de 2004 que las predisposiciones políticas predicen el consumo de información en las tertulias radiofónicas, la televisión por cable y las visitas a webs. Iyengar y Kahn (2009) concluyen que los individuos prefieren consumir información consistente con sus creencias previas. El experimento que realizaron mostrando noticias atribuidas a las cadenas de televisión Fox News, CNN, BBC y NPR demuestra que la polarización ideológica entre liberales y conservadores explica la selección de las noticas sobre política, relaciones raciales y la guerra de Irak en una u otra cadena. Los demócratas prefieren CNN y NPR, mientras que los republicanos prefieren Fox News (2009, pp. 3233). Mutz y Martin (2001) encontraron evidencias de que la gente prefiere aquellos medios que refuerzan sus opiniones frente a los que dan otra visión.

Las perspectivas actuales consideran la exposición selectiva como un predictor de los efectos de los medios. Entre estos efectos, la polarización es uno de los más presentes. La polarización política está irremediablemente unida a la exposición selectiva del consumo de medios, siendo posible que ambos puedan ser causa y consecuencia el uno del otro. La relación entre exposición selectiva, partidismo y polarización de las actitudes políticas ha sido corroborada por Mutz (2006). Stroud (2010) ha examinado cuál de los dos es causa del otro, encontrando que la exposición a medios afines contribuye a un mayor nivel de polarización. Goldman y Mutz (2011) comprobaron para 11 países que los ciudadanos manifiestan su favoritismo por los medios políticamente afines y que además este proceso es más fuerte en los países con un elevado paralelismo político. 


\section{El caso español}

Como hemos visto, el retorno a la teoría de la exposición selectiva en el contexto anglosajón se fundamenta en dos premisas: la multiplicación de canales para obtener información y la aparición de un modelo de periodismo alejado de la objetividad, que permite la exposición partidista. En el caso español, las premisas del modelo pluralista polarizado (Hallin y Mancini, 2004) conducen hacia un comportamiento partidista de las audiencias, ideal para examinar la existencia e intensidad del modelo de exposición selectiva entre la ciudadanía.

Hay una clara confirmación de que el periodismo político español está altamente politizado y que responde a alineamientos ideológicos con una creciente polarización (González y Novo, 2012; Martínez y Humanes, 2012; Van Dalen, De Vreese y Albaeck, 2012). Las razones de esta situación parecen ser básicamente dos. En primer término, las tensiones entre medios y partidos políticos que han producido las sucesivas concesiones de licencias en el sector audiovisual a partir de los años noventa y las ayudas a la prensa en sus distintas variantes (publicidad, subvenciones). En segundo término, la consolidación de un periodismo orientado al comentario, y vehiculado a través de las tertulias radiofónicas y televisivas, en el que los periodistas actúan como representantes de posiciones ideológicas coincidentes con las de los partidos políticos. El caso español se ajustaría a lo señalado por Mazzoleni (2010), cuando afirma que en algunos contextos el papel de adversario de los medios no se corresponde con la defensa de los intereses generales, sino que es una estrategia para actuar políticamente (2010, p. 99). "Otro dato revelador es que los ciudadanos perciben que el sesgo político de las noticias va en aumento. La tendencia a este respecto es claramente ascendente desde 2006, como demuestran los datos del estudio de la APM" (Farias y Roses, 2009, p. 21).

Los trabajos sobre exposición selectiva no han sido abundantes en España, aunque los que tenemos disponibles indican que sería necesario profundizar en este proceso y sus implicaciones. Los datos de la encuesta que se realizó en 1993 dentro del Comparative National Elections Project revelaron la existencia de paralelismo entre voto y lectura de prensa para 
los votantes del PSOE con el diario El País, los del PP con $A B C$ y los de IU con El País y El Mundo (Gunther, Montero y Wert, 1999, p. 36). Una década más tarde el trabajo de Alcalde Villacañas -aunque su objetivo era determinar la influencia del consumo de información en Antena 3 y TVE1 en el voto durante la campaña electoral de 1993- señaló que los votantes del PSOE son consumidores preferentemente de TVE1 y los del PP de Antena 3 (2003, p. 159).

Berganza y Martín Llaguno (2001) corroboraron la hipótesis de la exposición selectiva en la Comunidad Foral de Navarra durante las elecciones generales de 1996. A través de una encuesta a una muestra de 363 individuos encontraron relaciones de sintonía entre los votantes de Unión del Pueblo Navarro -conservador-y los periódicos El Mundo y Diario de Navarra y Antena 3 y Telecinco, mientras que para los votantes de PSOE e IU, los medios preferidos eran Diario de Noticias y El País junto a TVE1, Antena 3 y Telecinco (2001, p. 69).

Recientemente la Fundación Catdem ha publicado un informe sobre las vías de información de los diputados del Parlament de Catalunya. Los 99 diputados representantes de todos los partidos en el Parlament confirman con sus respuestas la hipótesis de la exposición selectiva partidista. Los resultados confirman con rotundidad la tesis del alto paralelismo entre medios y partidos políticos: las coincidencias a la hora de afiliar ideológicamente medios con partidos no bajan del $60 \%$ y en algunos casos alcanzan el 100\% (2012, p. 26). En segundo lugar, el consumo de información por parte de los diputados se ajusta a la hipótesis de la exposición selectiva, con excepción de la información en televisión, donde TV3 (la cadena de televisión pública en Catalunya) es la fuente principal para todos los encuestados (2012, pp. 60-61).

Roses (2010), en su estudio sobre confianza de la ciudadanía en el sistema de medios, aporta conclusiones valiosas para enmarcar nuestro trabajo. En primer lugar, confirmó que la identificación con la línea editorial de un medio de comunicación aumenta la credibilidad de la información (2010, p. 491). De manera inversa, la confianza en los medios se ve menos- 
cabada cuando el individuo se informa a través de varios de ellos (2010, p. 492). Por último, el grado de politización otorgado a las noticias obtiene una media de 7,5 puntos sobre 10 y ha ido creciendo a partir de 2008 (2010, p. 444); además

las expectativas de los ciudadanos son más bajas en cuanto a que "las informaciones de los medios sean imparciales" ( $M=3,12$; $D T=1,816)$ -una cuarta parte de los encuestados (percentil 25) deposita "muy poca" confianza en esto-; en que "los medios incluyan todos los puntos de vista en las informaciones que difunden" ( $M=3,57$; $D T=1,806)$ -una cuarta parte de los encuestados (percentil 25) deposita "poca" o "muy poca" confianza en esto (p.450).

Goldman y Mutz (2011), en el trabajo ya citado, incluyeron a España entre la muestra de países para su estudio sobre exposición transversal a la información (cross-cutting exposure), es decir, la exposición del individuo a puntos de vista políticos con los que no está de acuerdo, justo al anverso de la exposición selectiva. Sorprendentemente, los resultados alejan a nuestro país de los obtenidos por sus compañeros del modelo pluralista polarizado (Italia y Grecia), al revelar altos niveles de exposición transversal, cuando la tendencia general correlaciona negativamente paralelismo político y exposición.

\section{Metodología}

Desde el punto de vista metodológico, las investigaciones sobre exposición selectiva se han llevado a cabo bien a través de diseños experimentales, bien a través de encuestas (Stroud, 2006). Este hecho ha contribuido a la falta de consonancia en las conclusiones de los diferentes estudios, como hemos señalado anteriormente. Para Mutz y Martin (2001), los diseños de laboratorio están limitados al no poder reproducir las condiciones reales de elección de los contenidos. En la vida cotidiana los individuos pueden decidir exponerse (o no) a mensajes congruentes (o no) con sus opiniones. Por otra parte, los defensores de los estudios de laboratorio ponen en duda la tesis de la exposición selectiva argumentando que los resultados de estos trabajos no indican que la selección de la información sea un acto individual, sino que el motivo de la relación entre predisposiciones y 
exposición es el medio ambiente comunicativo - de facto selectivity - (Freedman y Sears, 1965).

Nuestro trabajo se enmarca dentro de los estudios basados en encuesta, es decir, intentamos examinar la hipótesis de la exposición selectiva en condiciones no controladas por el investigador. Se plantean dos objetivos principales: uno, corroborar empíricamente la exposición selectiva que realizan los españoles cuando se informan sobre la vida política en los periodos de campaña electoral, y dos, comprobar si este uso selectivo se corresponde con un comportamiento partidista de las audiencias, propio del modelo pluralista polarizado. Se han utilizado los datos correspondientes a los estudios poselectorales del Centro de Investigaciones Sociológicas (CIS) de 2008 (estudio 2757) y 2011 (estudio 2920). El primero se realizó entre el 12 de marzo y el 12 de mayo de 2008, con 6083 entrevistas. El segundo se realizó entre el 24 de noviembre de 2011 y el 15 de enero de 2012, con 6082 entrevistas. ${ }^{3}$ Estos dos estudios son especialmente valiosos para el trabajo que planteamos, puesto que se encuentran desagregados los datos sobre uso de cada uno de los diarios, emisoras de radio y cadenas de televisión mencionados por los encuestados como medio para informarse durante la campaña.

A partir de la literatura existente abordamos las siguientes hipótesis:

H1: Durante el periodo de campaña electoral se produce exposición selectiva de la información política guiada por las orientaciones políticas de la audiencia.

H2: La asociación entre exposición selectiva y orientaciones políticas es más fuerte en el uso de la prensa que en los medios audiovisuales.

H3: La selección de un medio para informarse se asocia con la elección de otros atendiendo a la proximidad ideológica entre los medios.

3 Más información sobre los dos estudios se puede consultar en: http://www.cis.es/cis/opencm/ES/1_encuestas/

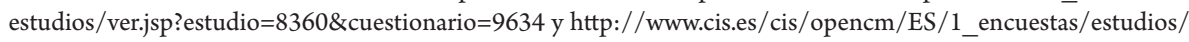
ver.jsp? estudio $=12604$. 
Las variables dependientes resultan de las tres preguntas de los dos estudios poselectorales sobre el medio por el cual se había seguido la campaña electoral: " $i Y$ qué periódico prefiere usted para seguir la información política y electoral?”; “iY qué cadena de televisión prefiere usted para seguir la información política y electoral?”; "¿Y qué emisora de radio prefiere usted para seguir la información política y electoral?”. Hay que indicar que estas preguntas no fueron contestadas por quienes habían respondido que nunca o casi nunca habían seguido la campaña por los medios (tabla 1). Finalmente se han utilizado para los análisis estadísticos sólo las respuestas para los medios (diarios, cadenas de televisión y de radio) con mayor audiencia según los datos del Estudio General de Medios, ${ }^{4}$ a los que se han sumado algunos medios que tienen importante presencia en sus comunidades autónomas (ver tablas 2, 3, y 4), o cuya línea editorial representa un espectro ideológico no cubierto por los otros medios (por ejemplo, La Gaceta o Intereconomía para el espectro más conservador). En el anexo se encuentran las características básicas de los medios de comunicación citados como fuente de información sobre las dos campañas electorales.

De acuerdo con las hipótesis planteadas se han considerado como variables independientes el recuerdo de voto en las elecciones generales correspondientes a cada uno de los estudios poselectorales, la cercanía a un partido político y la autoubicación ideológica (medida en la escala de 1-10, que se recodificó en cinco categorías). En la tabla 2 del anexo se encuentran las características ideológicas de los partidos políticos citados en las variables 'recuerdo de voto' y 'cercanía a un partido político.

Los datos se han sometido a análisis bivariados (tablas de contingencia y residuos tipificados corregidos) para testear las tres hipótesis. La prueba de contraste $\mathrm{X}^{2}$ permite comprobar si existen asociaciones estadísticamente significativas entre las variables. Los valores z (residuos tipificados corregidos) muestran qué casillas de la tabla de contingencia son las responsables de la asociación. En este caso se consideraron estadísticamente significativos los residuos con valores $\geq 1,96$. En las tablas 2 a 6 se ofrecen los valores z en lugar de los porcentajes o frecuencias.

4 http://www.aimc.es/-Datos-EGM-Resumen-General-.html. 


\section{Resultados}

La televisión es el medio de comunicación preferente para obtener información política. Si en 2008 el 53,5\% de los encuestados declaraba haber seguido la campaña a través de la televisión todos o casi todos los días, en 2011 el porcentaje había aumentado (tabla 1). Casi la mitad de las muestras en ambos años manifiestan no leer nunca o casi nunca los periódicos y más del $60 \%$ no escucha ninguna emisora de radio para informarse durante la campaña electoral. Más concretamente, los informativos de la cadena pública TVE y los de Antena 3 suman el 57\% del consumo en los dos momentos considerados.

\section{Tabla 1 \\ Consumo de información política y electoral durante campaña electoral (\%)}

\begin{tabular}{|l|c|c|c|c|c|c|}
\hline & \multicolumn{3}{|c|}{2008} & \multicolumn{3}{c|}{2011} \\
\hline & Prensa & Radio & Televisión & Prensa & Radio & Televisión \\
\hline Todos o casi todos los días & 22,2 & 19,2 & 53,5 & 22,5 & 18,6 & 56,6 \\
\hline Cuatro o cinco días por semana & 5,5 & 4,7 & 10,3 & 6,1 & 3,9 & 10,1 \\
\hline Dos o tres días por semana & 7,8 & 4,2 & 9,0 & 7,8 & 4 & 8,6 \\
\hline Sólo los fines de semana & 4,9 & 1,1 & 1,9 & 4 & 1,1 & 1,5 \\
\hline De vez en cuando & 10,9 & 8,8 & 11,1 & 10,5 & 8,2 & 9,7 \\
\hline Nunca o casi nunca & 48,2 & 61,1 & 13,9 & 48,7 & 63 & 13,2 \\
\hline N. C. &, 4 &, 9 &, 2 & 0,5 & 1,3 & 0,7 \\
\hline Total & 100 & 100 & 100 & 100 & 100 & 100 \\
\hline
\end{tabular}

Fuente: CIS. Elaboración propia.

Los datos corroboran la tendencia general de consumo de información fuera de los periodos electorales. Según datos del CIS (barómetro de mayo de 2010), la mayor parte de los ciudadanos no sólo se informa básicamente por la televisión (46,2\%), sino que además lo hace por un único canal $(59,2 \%)$. Esta es la tendencia general en el consumo de información en España, donde predomina la televisión con un índice de penetración superior al $88 \%$ frente a los diarios $(32,4 \%)$ y la radio $(61,5 \%)$, según los últimos datos del Estudio General de Medios (noviembre de 2013). 


\section{Exposición selectiva y orientaciones políticas}

En el sistema de medios español, caracterizado por el alto nivel de paralelismo político y polarización (Hallin y Mancini 2004), debería encontrarse también un alto partidismo de las audiencias. Para que se produzca este fenómeno, en primer lugar, los ciudadanos habrán de ser capaces de situar ideológicamente cada medio de comunicación. En el gráfico 1 se observa cómo los encuestados en el Informe de la profesión periodística en Espa$\tilde{n} a(2010$, p. 117) ubicaban cada medio en una escala de 1 (izquierda) a 10 (derecha). Los datos reflejan que las puntuaciones medias forman tres grandes grupos de izquierda y centro-izquierda (RNE, TVE, El País, Ser y La Sexta), centro-derecha (Onda Cero, Antena 3 y Telecinco) y derecha ( $E l$ Mundo, Abc, La Razón y COPE).

\section{Gráfico 1 \\ Ubicación ideológica de los medios según la ciudadanía}

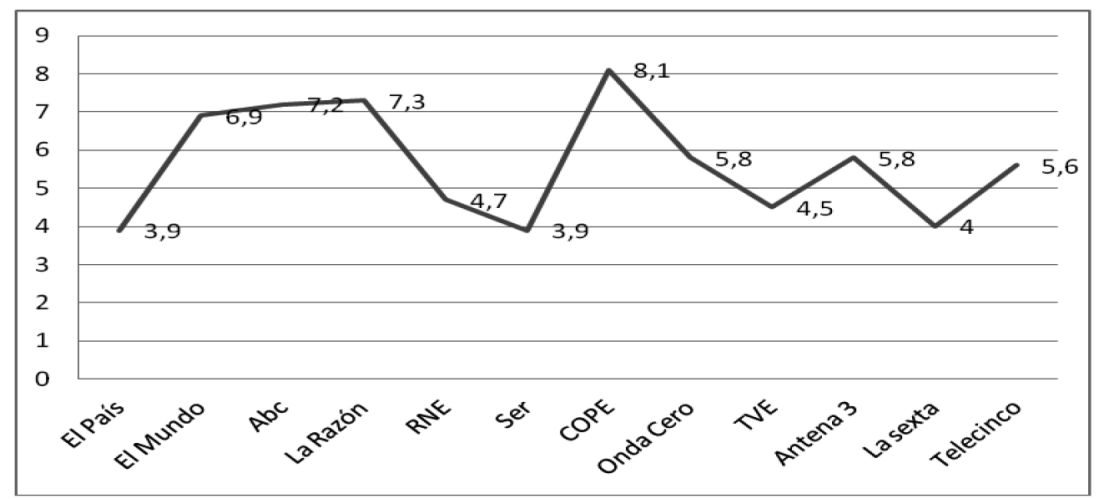

Fuente: Informe de la profesión periodística en España (2010).

A partir de esta premisa se aborda la primera hipótesis del estudio sobre la relación entre exposición selectiva y orientaciones políticas. De manera general los análisis bivariados corroboran que en 2008 la elección de diarios, cadenas de televisión y emisoras de radio se asocia de manera estadísticamente significativa $(\mathrm{p} \leq 0,000)$ con las tres variables independientes: la ideología, el recuerdo de voto y la cercanía a un partido político. En 2011 se reproducen las mismas asociaciones estadísticamente significativas. La fuerza de las asociaciones medida a través de la V de Cramer muestra valo- 
res moderados y distintos para cada una de las variables independientes: el recuerdo de voto y la cercanía a un partido obtienen medidas de asociación más altas que la autoubicación ideológica (tablas 2, 3 y 4) en los tres tipos de medios de comunicación. Sí existe coincidencia en el aumento de los valores de las medidas de asociación en 2011 respecto a 2008. El análisis de los residuos tipificados corregidos nos ha permitido profundizar en la asociación entre las orientaciones políticas y el consumo selectivo de medios.

La prensa es el medio de información política y electoral más condicionado por el efecto de la exposición selectiva provocada por las orientaciones políticas. Los valores de la $\mathrm{V}$ de Cramer son siempre más altos que en las asociaciones de las variables independientes con el consumo de radio y de televisión. Por otra parte, la relación es más fuerte con el recuerdo de voto $(0,424$ y 0,429$)$ y la cercanía a un partido político $(0,428$ y 0,435$)$ que con la ideología ( 0,276 y 0,302). Los valores de los residuos permiten concluir que la adscripción a un partido político y la ideología correrían en paralelo a la lectura del periódico que mejor 'representa' a ese partido y tendencia. Se encuentra una excepción -El Periódico- cuyos lectores manifiestan una ideología de centro-izquierda e izquierda, pero la conducta de voto y de simpatía hacia un partido político se corresponde con una organización de centro derecha (CIU), sobre todo en 2011.

El uso de los medios audiovisuales, aunque también es coherente con el proceso de exposición selectiva, muestra resultados menos estables que los obtenidos para el consumo de diarios. Como ya se ha señalado, los valores de la V de Cramer (tablas 3 y 4) siempre están por debajo de 0,400, lo cual indica que la asociación entre las variables independientes y el uso de la radio y la televisión para informarse es más débil.

Para el consumo de televisión, en primer lugar, hay que señalar un mayor número de residuos que no son estadísticamente significativos, concretamente para la variable 'ideología', lo cual indica menos ajuste a la hipótesis de la exposición selectiva, aunque se descartaría la hipótesis nula de igualdad en la distribución. 


\section{Tabla 2}

\section{Exposición selectiva de la lectura de prensa (residuos tipificados corregidos) $^{5}$}

\begin{tabular}{|c|c|c|c|c|c|c|}
\hline & \multicolumn{3}{|c|}{2008} & \multicolumn{3}{|c|}{2011} \\
\hline & Ideología $^{6}$ & Voto & Cercanía & Ideología & Voto & Cercanía \\
\hline El País & $9,3(2)$ & 13,4 (PSOE) & 10,6 (PSOE) & $12,4(2)$ & 13,3 (PSOE) & 9,7 (PSOE) \\
\hline El Mundo & $11(4)$ & $17,9(\mathrm{PP})$ & $16,9(\mathrm{PP})$ & $7,9(4)$ & $10,9(\mathrm{PP})$ & $9,5(\mathrm{PP})$ \\
\hline $\mathrm{Abc}$ & $6,8(5)$ & $8,3(\mathrm{PP})$ & $10,4(\mathrm{PP})$ & $6,9(4)$ & 7,6 (PP) & $8,5(\mathrm{PP})$ \\
\hline La Razón & $4,7(4)$ & 6,6 (PP) & $5,6(\mathrm{PP})$ & $2,9(5)$ & 4,2 (PP) & $2,4(\mathrm{PP})$ \\
\hline Público & $5,3(1)$ & 3,6(IU) & 4,6 (IU) & $8,2(1)$ & $11,1(\mathrm{IU})$ & $10,5(\mathrm{IU})$ \\
\hline La Vanguardia & $3,2(3)$ & 9,9 (CIU) & 10,6 (CIU) & * & 18,4 (CIU) & 16,2 (CIU) \\
\hline El Periódico & $2,9(2)$ & 9,5 (CIU) & $\begin{array}{l}6,7(\mathrm{CiU}) \\
6,5(\mathrm{ERC})\end{array}$ & $2,3(1)$ & $9(\mathrm{CIU})$ & 6,8 (CIU) \\
\hline La Voz de Galicia & * & $16,2(\mathrm{BNG})$ & 14,3 (BNG) & $2,5(3)$ & 12,9 (BNG) & 11,3 (BNG) \\
\hline El Correo & $1,9(3)$ & 11,7 (PNV) & $11,4(\mathrm{PNV})$ & $2,2,(2)$ & $12,2(\mathrm{PNV})$ & $12,4(\mathrm{PNV})$ \\
\hline La Gaceta & - & - & - & $4,4(5)$ & 4,8 (PP) & $5,7(\mathrm{PP})$ \\
\hline $\mathrm{X} 2(\mathrm{p} \leq .000)$ & 896,460 & 4860,198 & 3636,690 & 909,455 & 5013,748 & 3625,919 \\
\hline V de Cramer & 0,276 & 0,424 & 0,428 & 0,302 & 0,429 & 0,435 \\
\hline
\end{tabular}

* Los residuos no son estadísticamente significativos.

Fuente: CIS. Elaboración propia.

Pero el resultado más destacado es la aparición de cambios en la selección de la cadena de televisión en los dos momentos. El más evidente es el caso de Cuatro, que tras su compra a Prisa por Mediaset en 2010 varió su línea informativa, lo que se ha reflejado en cambios de su audiencia. En 2008 los residuos más altos se concentraban en una única casilla: votantes del PSOE, cercanos a IU y de centro-izquierda. En 2011, los residuos se acercan a no ser estadísticamente significativos, y además para la variable 'cercanía a un partido' alcanzan el mismo valor para IU, Amaiur y UPyD. En La Sexta se ha producido un desplazamiento hacia un tipo de telespectador situado más hacia la izquierda ideológica y política (los residuos han incrementado su valor). La 2 sería el ejemplo del medio cuya audiencia no estaría orientada por la exposición selectiva motivada políticamente: los

\footnotetext{
5 Se muestra en cada casilla el valor del residuo más alto en el cruce de las dos variables.

6 La variable 'autoubicación ideológica' se ha recodificado, a partir de la escala 1-10, en cinco categorías: 1 = izquierda, $2=$ centro izquierda, $3=$ centro, $4=$ centro derecha y $5=$ derecha.
} 
residuos o no son significativos o son muy pequeños. Por último, la televisión es el único de los tres medios en el que la asociación entre el recuerdo de voto y la cercanía a un partido son ligeramente más débiles en 2011 que cuatro años antes.

Tabla 3

\section{Exposición selectiva y consumo de televisión (residuos tipificados corregidos)}

\begin{tabular}{|c|c|c|c|c|c|c|}
\hline & \multicolumn{3}{|c|}{2008} & \multicolumn{3}{|c|}{2011} \\
\hline & Ideología & Voto & Cercanía & Ideología & Voto & Cercanía \\
\hline TVE 1 & $2,4(2)$ & 7,3 (PSOE) & 7,8 (PSOE) & $5,1(2)$ & 7,7 (PSOE) & 8 (PSOE) \\
\hline La 2 & * & $\begin{array}{c}2,6 \text { (IU) } \\
2,7 \text { (UPyD) }\end{array}$ & $3,2(\mathrm{CC})$ & * & * & 4,5 (Gero-BAI) \\
\hline Antena 3 & $10,4(4)$ & 16,4 (PP) & 14,3 (PP) & $6,3(3)$ & $10,9(\mathrm{PP})$ & 11,8 (PP) \\
\hline Cuatro & $7(2)$ & 7,9 (PSOE) & 6,3 (IU) & $\begin{array}{l}2,2(1) \\
2,2(2)\end{array}$ & 3,6 (IU) & $\begin{array}{c}2,8(\mathrm{IU}) \\
2,5(\mathrm{UPyD}) \\
2,5 \text { (AMAIUR) }\end{array}$ \\
\hline Telecinco & * & 4,7 (PSOE) & 4,1 (PSOE) & * & 3,1 (PSOE) & 4,7 (PSOE) \\
\hline La Sexta & $2,4(2)$ & 3,3 (PSOE) & 2,5 (PSOE) & $3,9(1)$ & 6,8 (PSOE) & 4,8 (IU) \\
\hline Canal Sur & $5,7(2)$ & 5,6 (PSOE) & 4,1 (PSOE) & $5,1(2)$ & 5 (PSOE) & 5,4 (PSOE) \\
\hline TV3 & $3,6(2)$ & $27,1(\mathrm{CIU})$ & 24,8 (CIU) & $5,5(1)$ & 33 (CIU) & 24,8 (CIU) \\
\hline Canal Nou & $4,9(5)$ & 6,7 (PP) & 6,2 (PP) & $4,1(5)$ & 4,5 (PP) & 3,9 (PP) \\
\hline Telemadrid & $6,3(4)$ & 9,5 (PP) & 10,4 (PP) & $4(4)$ & 4,5 (PP) & 4,6 (PP) \\
\hline TVG & * & 11,4 (BNG) & $11,1(\mathrm{BNG})$ & $2,2(4)$ & $5,3(\mathrm{BNG})$ & 3,6 (BNG) \\
\hline ETB1 & * & 28,3 (PNV) & 24,1 (PNV) & $3,9(1)$ & 28,5 (AMAIUR) & 21,9 (PNV) \\
\hline $\begin{array}{c}\text { Intereconomía } \\
\text { TV }\end{array}$ & - & - & - & $9,8(5)$ & 10,4 (PP) & 10,7 (PP) \\
\hline $\mathrm{X} 2(\mathrm{p} \leq .000)$ & 602,915 & 4875,470 & 3792,686 & 585,848 & 4692,873 & 3266,441 \\
\hline V de Cramer & 0,181 & 0,336 & 0,356 & 0,184 & 0,313 & 0,320 \\
\hline
\end{tabular}

* Los residuos no son estadísticamente significativos.

Fuente: CIS. Elaboración propia.

Las emisoras de radio se asemejan más en la tendencia hacia la exposición selectiva a la prensa escrita que a la televisión (tabla 4). Aunque los valores de la $\mathrm{V}$ de Cramer son más bajos, también se han incrementado en 2011 para el recuerdo de voto y la cercanía a un partido. Salvo en el caso de RAC1 (propiedad del mismo grupo que La Vanguardia), el resto de las emisoras mantienen una audiencia con las mismas orientaciones políticas. 


\section{Tabla 4}

\section{Exposición selectiva y consumo de radio (residuos tipificados corregidos)}

\begin{tabular}{|c|c|c|c|c|c|c|}
\hline & \multicolumn{3}{|c|}{2008} & \multicolumn{3}{|c|}{2011} \\
\hline & Ideología & Voto & Cercanía & Ideología & Voto & Cercanía \\
\hline Ser & $9,7(2)$ & 16,5 (PSOE) & 15,1 (PSOE) & $10,7(2)$ & 15 (PSOE) & 13,7 (PSOE) \\
\hline COPE & $15,6(4)$ & 19,3 (PP) & $18,6(\mathrm{PP})$ & $8,2(4)$ & $12,5(\mathrm{PP})$ & $12,8(\mathrm{PP})$ \\
\hline Onda Cero & $8,2(3)$ & $11,8(\mathrm{PP})$ & $10,3(\mathrm{PP})$ & $4,5(4)$ & $9,7(\mathrm{PP})$ & $7,3(\mathrm{PP})$ \\
\hline RNE & * & 2,6 (PSOE) & 2,9 (PSOE) & * & $2,3(\mathrm{IU})$ & 2,1 (PSOE) \\
\hline Catalunya Radio & $2,1(2)$ & $19,1(\mathrm{CIU})$ & $17,9(\mathrm{CIU})$ & $3,8(1)$ & 15 (CIU) & $8,6(\mathrm{CIU})$ \\
\hline Punto Radio & $2,8(3)$ & $1,9(\mathrm{PP})$ & 2,6 (PP) & $2,7(3)$ & 2,1 (PP) & $3(\mathrm{PP})$ \\
\hline RAC 1 & $2(2)$ & 12,4 (ECR) & 13,6 (ERC) & * & 16,3 (CIU) & $12,8(\mathrm{CIU})$ \\
\hline $\begin{array}{c}\text { Radio } \\
\text { Intereconomía }\end{array}$ & - & - & - & $4,6(4)$ & 4,4 (PP) & 3,8 (PP) \\
\hline $\mathrm{X} 2$ & 617,035 & 2449,034 & 2191,381 & 388,378 & 1989,467 & 1605,134 \\
\hline V de Cramer & 0,272 & 0,356 & 0,386 & 0,243 & 0,374 & 0,399 \\
\hline
\end{tabular}

* Los residuos no son estadísticamente significativos.

Fuente: CIS. Elaboración propia .

\section{Consonancia de la exposición selectiva entre medios}

En la hipótesis 3 se propone que el uso de un determinado medio de comunicación está asociado con el consumo de otros medios con una línea editorial próxima. Esta proximidad puede deberse a dos causas: compartir una misma ideología o ser parte de un mismo grupo mediático. Los resultados muestran ambas situaciones (tabla 5 y 6 ).

La lectura selectiva de periódicos presenta dos tendencias en función del ámbito de difusión. Los residuos para los diarios estatales se acumulan bien por proximidad ideológica, bien por pertenencia al mismo grupo. En el primer supuesto estarían los diarios El Mundo, Abc, La Razón, que a pesar de pertenecer a las mismas empresas que otras cadenas de radio y televisión, su uso como fuente de información se asocia a los medios detectados como más próximos ideológicamente. Es más evidente para los lectores de La Razón que, pudiendo completar la visión del diario con sus partners Onda Cero y Antena 3, no lo hacen. La influencia del efecto del grupo mediático de pertenencia está presente en Público y La Gaceta: en ambos casos se 


\section{Tabla 5}

\section{Relación entre consumo de prensa y medios audiovisuales (residuos tipificados corregidos)}

\begin{tabular}{|c|c|c|c|c|}
\hline Diario & \multicolumn{2}{|c|}{2008} & \multicolumn{2}{c|}{2011} \\
\hline & Emisora de radio & Cadena de televisión & Emisora de radio & Cadena de televisión \\
\hline El País & $12,8($ Ser) & 11,2 (Cuatro) & 10,4 (Ser) & 8,5 (TVE) \\
\hline El Mundo & 11,9 (Onda Cero) & 10,5 (Antena 3) & 7,7 (Onda Cero) & 6,3 (Antena 3) \\
\hline Abc & 4,7 (Punto Radio) & 6,9 (Telemadrid) & 6,3 (COPE) & 5,6 (Telemadrid) \\
\hline La Razón & 5,7 (Radio España) & 9,4 (Telemadrid) & $\begin{array}{c}3,4 \text { (Radio } \\
\text { Intereconomía) }\end{array}$ & 2,6 (Telemadrid) \\
\hline Público & 2,2 (Ser) & $5,5($ La Sexta) & 2,2 (Ser) & 5,5 (La Sexta) \\
\hline La Vanguardia & 16,7 (Catalunya Radio) & $17,5(\mathrm{TV} 3)$ & 13,5 (RAC1) & 20,3 (TV3) \\
\hline El Periódico & 6,6 (Catalunya Radio) & $18,1(\mathrm{TV} 3)$ & 9,6 (Catalunya Radio) & 16,3 (TV3) \\
\hline La Voz de Galicia & 15,7 (Radio Galega) & $9,2(\mathrm{TVG})$ & 12 (Radio Galega) & 9,7 (TVG) \\
\hline El Correo & 8,2 (Radio Euskadi) & $9,2(\mathrm{ETB} 2)$ & 5,6 (Radio Euskadi) & 10,7 (ETB2) \\
\hline La Gaceta & - & - & 4 (COPE) & 26,7 (Intereconomía) \\
\hline X2 & 2978,484 & 5490,584 & 2320,184 & 6457,395 \\
\hline V de Cramer & 0,394 & 0,309 & 0,441 & 0,360 \\
\hline
\end{tabular}

Fuente: CIS. Elaboración propia.

reconoce que la cadena de televisión más afín es la del mismo grupo. En el caso de El País, en 2008, su uso respecto a los otros medios estaría basado en la pertenencia al grupo Prisa, mientras que en 2011 sus lectores se desplazan desde Cuatro a TVE1, en un comportamiento similar al que se ha reseñado anteriormente para Cuatro. El uso de los diarios de ámbito autonómico se asocia con la exposición a radios y televisiones de la misma cobertura territorial, aunque no siempre estén cercanos ideológicamente. La proximidad de la información ofrecida por los tres tipos de medios podría ser una explicación razonable.

Entre los medios audiovisuales la vinculación con el grupo mediático se presenta en la mayoría de los casos como motivo de la selección entre ellos. Sólo los oyentes de las emisoras que no participan en la propiedad de cadenas de televisión acuden para informarse a una cadena ideológicamente cercana. Así sucede para la COPE en los dos años analizados, y para la Ser en 2011 tras la venta de Cuatro a Mediaset, como hemos indicado. 


\section{Tabla 6}

\section{Relación del consumo de radio y televisión (residuos tipificados corregidos)}

\begin{tabular}{|c|c|c|}
\hline Emisora de radio & 2008 & 2011 \\
\hline Ser & Cadena de televisión & Cadena de televisión \\
\hline COPE & 12,5 (Cuatro) & 4,2 (TVE1) \\
\hline Onda Cero & 7,6 (Antena 3) & 6,5 (Intereconomía TV) \\
\hline RNE & 11,8 (Antena 3) & 10,7 (Antena 3) \\
\hline Catalunya Radio & 7,1 (TVE1) & 18,7 (TVE1) \\
\hline Punto Radio & 29,4 (TV3) & 3,7 (Telecinco) \\
\hline RAC 1 & 6 (Telemadrid) & 21,9 (TV3) \\
\hline Radio Intereconomía & $15($ TV3) & 7,3 (Intereconomía TV) \\
\hline X2 & - & 2506,095 \\
\hline V de Cramer & 3174,628 & 0,394 \\
\hline
\end{tabular}

Fuente: CIS. Elaboración propia.

\section{Conclusiones}

Los resultados del trabajo nos permiten corroborar la existencia de exposición selectiva orientada por las predisposiciones políticas durante los dos periodos de campaña electoral y por lo tanto se ha confirmado la idea de que al sistema de medios español le corresponden audiencias partidistas (Hallin y Mancini, 2004). Esta asociación es más fuerte para las dos variables referidas a la identificación con un partido político que para la ideología. Ello indicaría que el paralelismo político en España se hace más visible en la afinidad entre medios de comunicación y partidos políticos, y así lo percibe la ciudadanía. Se necesitarían más estudios sobre consumo de información en periodo no electoral para saber si la relación entre orientaciones políticas y uso de los medios se manifiesta de igual forma. Como han señalado diferentes trabajos, la información política en España gira en torno a la actividad de los partidos políticos (Strömback y Luengo, 2008; Humanes, 2011; Martínez y Humanes, 2012).

Por otra parte se ha demostrado que el tipo de medio (prensa, radio o televisión) afecta la intensidad de la exposición selectiva, siendo la prensa el medio que ofrece asociaciones más fuertes entre orientaciones políti- 
cas y selección partidista. Este dato manifiesta que la naturaleza comercial de la información audiovisual en España beneficiaría un menor grado de paralelismo político, y la mayoría de las empresas aún no habrían asumido como estrategia de negocio el partidismo político (al menos en el momento de realizar el trabajo de campo). No obstante, una de las limitaciones del trabajo se encuentra precisamente en la no disponibilidad de información sobre los tipos de programas (informativos, tertulias, infoentretenimiento) consumidos por los encuestados. En este sentido, para posteriores investigaciones también habría que considerar los medios vehiculados a través de la red como fuente de información política para testear si en el caso español se reproducen las mismas tendencias hacia la polarización que se han encontrado en el contexto norteamericano.

Los resultados revelan cómo los individuos cambian su fuente de información cuando detectan que la línea editorial del medio de comunicación se ha modificado. Así se ha revelado sobre todo en el caso de la cadena de televisión Cuatro. Los gestores de las empresas de medios pueden encontrar valiosas las conclusiones de este trabajo por cuanto confirman que las predisposiciones políticas intervienen en la fidelización de nichos de audiencia.

No obstante la confirmación de las hipótesis que se habían planteado, conviene no olvidar la necesidad de investigar el proceso de exposición selectiva a través de modelos multivariables que permitan calibrar el peso de las tres variables que se han considerado en este trabajo. Por ejemplo, hay que explorar el conocimiento, el interés y el nivel de participación como predictores del consumo selectivo de medios.

Aunque esta investigación no analiza las cuestiones relacionadas con la credibilidad y confianza en los medios, sí se puede aventurar que el reconocimiento por parte de la ciudadanía de sesgos políticos en los contenidos periodísticos pudiera estar asociado a una mayor credibilidad para los medios afines. Por el contrario -y siguiendo la hipótesis de la hostilidadsería una explicación del descrédito de los medios que muestran perspectivas distintas. 
Los resultados del estudio nos permiten avanzar el interés por analizar en posteriores trabajos si la interacción entre las orientaciones políticas y el uso partidista de los medios son predictores de la polarización política, la falta de deliberación y entendimiento de las opiniones contrarias. Se estaría dando luz a los debates sobre el estado de la calidad de nuestro sistema democrático, la contribución de los medios al mismo y la responsabilidad de la ciudadanía.

\section{Referencias}

Alcalde Villacampa, J. (2003). "Medios de comunicación como intermediarios del voto: la influencia de TVE1 y Antena 3 en las elecciones legislativas de 1993”. En: REIS, 104 (3), pp. 145-178.

Arceneaux, K., Johnson, M. y Murphy, C. (2012). "Polarized political communication, oppositional media hostility, and selective exposure". En: The Journal of Politics, 74 (1), pp. 174-186.

Bennett, W. L. y Iyengar, S. (2008). "A New Era of Minimal Effects? The Changing Foundations of Political Communication”. En: Journal of Communication, 58 (4), pp. 707-731.

Bryant, J. y Miron, T, (2004). “Theory and Research in Mass Communication”. En: Journal of Communication, 54, pp. 662-704.

D’Alessio, D. y Allen, M. (2002). "Selective exposure and dissonance after decisions”. En: Psychological Reports, 91(2), 527-532.

Dylko, I. y McCluskey, M. (2012). "Media Effects in an Era of Rapid Technological Transformation: A Case of User-Generated Content and Political Participation”. En: Communication Theory, 22 (3), pp. 250-278.

Farias, P. et al. (2010). Informe Anual de la Profesión Periodística 2010. Madrid: APM. 
Farias, P. y Roses, S. (2009). "La crisis acelera el cambio del negocio informativo". En: Estudios sobre el mensaje periodístico, 15, pp.15-32.

Freedman, J. y Sears, D. (1965). “Selective exposure”. En: Berkowitz, L. (ed.). Advances in experimental social psychology (pp. 57-97). Nueva York: Academic Press.

Frey, D. (1986). "Recent research on selective exposure to information". En: Berkowitz, L. (ed.). Advances in experimental social psychology (pp. 41-80). Nueva York: Academic Press.

Cano i Castells, F. y Rodon i Casarramona, A. (2012). La informació al parlament de Catalunya. Barcelona: CatDem.

Goldman, S. K. y Mutz, D. C. (2011). “The friendly media phenomenon: A cross-national analysis of cross-cutting exposure”. En: Political Communication, 28, pp. 42-66.

Gunther, R., Montero, J. R. y Wert, J. I. (1999). “The media and politics in Spain: from dictatorship to democracy”. En: WP no 176, Institut de Ciencies Polítiques i Socials.

Hallin, D. y Mancini, P. (2004). Comparing Media Systems. Three models of media and politics. Nueva York: Cambridge University Press.

Humanes (2011). "Nuevos temas noticiosos en las elecciones generales de 2004. La presencia de metacobertura en la prensa española". En: Zer-Revista de Estudios de Comunicación, 14 (26), pp. 105-128.

Humanes y de los Ángeles Moreno, M. (2012). "El efecto agenda sobre los temas de campaña en las elecciones generales de 2008”. En: adComunica, 3, pp.: 191-207.

Iyengar, S. y Kahn, K. (2009). "Red media, blue media: evidence of ideological selectivity in media use”. En: Journal of Communication, 59, pp. 19-39. 
Iyengar, S. et al. (2008). "Selective exposure to campaign communication:

The role of anticipated agreement and issue public membership". En: Journal of Politics, 70, pp. 186-200.

Kappler, J. (1960). The effects of mass communication. Toronto: The Free Press.

Kinder, D. (2003). "Communication and politics in the age of information". En: Oxford hand book of political psychology, pp. 357-393. Oxford: Oxford University Press.

Lazarsfeld, P., Berelson, B. y Gaudet, H. (1948). The people's choice. Nueva York: Columbia University Press.

Martín Llaguno, M. y Berganza, R. (2001). “Votantes y medios de comunicación en las elecciones nacionales españolas de 1996: ¿exposición selectiva o influencia mediática?”. En: Comunicación y Sociedad, 1, pp. 51-70.

Martínez Nicolás, M. y Humanes, M. L. (2012). “Culturas profesionales del periodismo político en España. El discurso de los periodistas sobre la política y las funciones políticas de los medios”. En: Casero, A. (coord.). Periodismo político en España: concepciones, tensiones y elecciones (pp. 47-66). La Laguna: Cuadernos Artesanos de Latina, 33.

Matthes, J. (2012). "Exposure to counterattitudinal news coverage and the timing of voting decisions”. En: Communication Research, 39 (2), pp. 147-169.

Mazzoleni, G. (2010). La comunicación política. Madrid: Anaya.

Mutz, D. (2006). Hearing the other side: Deliberative versus participatory democracy. Nueva York: Cambridge University Press. 
Mutz, D. y Martin, P. (2001). "Facilitating communication across lines of political difference: The role of mass media”. En: American Political Science Review, 95, pp. 97-114.

Pfau, M. W., Houston, J. B. y Semmler, S. M. (2007). Mediating the vote: The changing media landscape in US presidential campaigns. Maryland: Rowman \& Littlefield.

Strömbäck, J. y Luengo, Ó. G. (2008). “Polarized Pluralist and Democratic Corporatist Models A Comparison of Election News Coverage in Spain and Sweden”. En: International Communication Gazette, 70 (6), pp. 547-562.

Stroud, N. (2006). "Selective exposure to partisan information” Ph. D. dissertation, University of Pennsylvania. Disponible en: http://repository.upenn.edu/dissertations/AAI3246247 [fecha de consulta: 12 de noviembre de 2012].

Stroud, N. (2008). "Media use and political predispositions: Revisiting the concept of selective exposure". En: Political Behavior, 30, pp. 341-366.

Stroud, N. (2010). "Polarization and partisan selective exposure". En: Journal of Communication, 60, pp. 556-576.

Stroud, N. (2011). Niche News: The Politics of News Choice: The Politics of News Choice. Oxford: Oxford University Press.

Webster, J. (2005). "Beneath the Veneer of Fragmentation: Television Audience Polarization in a Multichannel World”. En: Journal of Communication, 55, pp. 366-382.

Valentino, N. et al. (2009). "Selective exposure in the Internet age: The interaction between anxiety and information utility". En: Political Psychology, 30 (4), pp. 591-613. 
Vallone, R. P., Ross, L. y Lepper, M. R. (1985). “The hostile media phenomenon: Biased perception and perceptions of media bias in coverage of the Beirut massacre". En: Journal of Personality and Social Psychology, 49 (3), pp. 577-585.

Van Dalen, A., De Vreese, C. H. y Albæk, E. (2012). “Different roles, different content? A four-country comparison of the role conceptions and reporting style of political journalists”. En: Journalism, 13 (7), pp. 903-922.

Zaller, J. (1992). The nature and origins of mass opinion. Nueva York: Cambridge University Press. 


\section{Anexos}

\section{Tabla 1}

\section{Características de los medios de comunicación correspondientes a las variables dependientes}

\begin{tabular}{|c|c|c|c|}
\hline Medio & Propiedad & Grupo mediático & Ámbito geográfico \\
\hline El País & Privada & Prisa & Estatal \\
\hline El Mundo & Privada & Unidad editorial & Estatal \\
\hline $\mathrm{Abc}$ & Privada & Vocento & Estatal \\
\hline La Razón & Privada & A3media & Estatal \\
\hline Público & Privada & Display Connectors & Estatal \\
\hline La Vanguardia & Privada & Grupo Godó & Comunidad Autónoma-Regional-Catalunya \\
\hline El Periódico & Privada & Grupo Z & Comunidad Autónoma-Regional-Catalunya \\
\hline La Voz de Galicia & Privada & Corporación Voz de Galicia & Comunidad Autónoma-Regional-Galicia \\
\hline El Correo & Privada & Vocento & Comunidad Autónoma-Regional-País Vasco \\
\hline La Gaceta & Privada & Grupo Intereconomía & Estatal \\
\hline RNE & Pública & - & Estatal \\
\hline Ser & Privada & Prisa & Estatal \\
\hline COPE & Privada & Conferencia Episcopal & Estatal \\
\hline Onda Cero & Privada & A3media & Estatal \\
\hline Catalunya Radio & Pública & - & Comunidad Autónoma-Regional-Catalunya \\
\hline Punto Radio & Privada & Vocento & Estatal \\
\hline RAC 1 & Privada & Grupo Godó & Comunidad Autónoma-Regional-Catalunya \\
\hline Radio Intereconomía & Privada & Grupo Intereconomía & Estatal \\
\hline TVE & Pública & - & Estatal \\
\hline Telemadrid & Pública & - & Comunidad Autónoma-Regional-Madrid \\
\hline Antena 3 & Privada & A3media & Estatal \\
\hline La sexta & Privada & A3media & Estatal \\
\hline Telecinco & Privada & Mediaset & Estatal \\
\hline TV3 & Pública & - & Comunidad Autónoma-Regional-Catalunya \\
\hline Canal Nou & Pública & - & Comunidad Autónoma-Regional-Valencia \\
\hline TVG & Pública & - & Comunidad Autónoma-Regional-Galicia \\
\hline ETB1 & Pública & - & Comunidad Autónoma-Regional-País Vasco \\
\hline Canal Sur & Pública & - & Comunidad Autónoma-Regional-Andalucía \\
\hline Intereconomía TV & Privada & Grupo Intereconomía & Estatal \\
\hline
\end{tabular}




\section{Tabla 2}

Partidos políticos correspondientes a las variables 'recuerdo de voto' y 'cercanía a un partido político'

\begin{tabular}{|c|c|}
\hline Partido Político & Ideología/orientación política \\
\hline Partido Popular (PP) & Conservador \\
\hline Partido Socialista Obrero Español (PSOE) & Socialdemócrata \\
\hline Izquierda Unida (IU) & Izquierda/Ecologista \\
\hline Convergencia i Unió (CIU) & Conservador/Nacionalista \\
\hline Esquerra Republicana de Catalunya (ERC) & Izquierda/Nacionalista \\
\hline Partido Nacionalista Vasco (PNV) & Conservador/Nacionalista \\
\hline Unión, Progreso y Democracia (UPy) & "Nacional, progresista y transversal” \\
\hline Bloque Nacionalista Galego (BNG) & Izquierda/Nacionalista \\
\hline Amaiur & Izquierda/Nacionalista \\
\hline
\end{tabular}

